\title{
MRI of Perfusion-Diffusion Mismatch in Non-Human Primate (Baboon) Stroke: A Preliminary Report
}

\author{
Hsiao-Ying Wey ${ }^{1,2}$, Ghazwan M. Kroma ${ }^{2}$, Jinqi Li ${ }^{1,2}$, M. Michelle Leland ${ }^{5}$, Lisa Jones ${ }^{5}$ and \\ Timothy Q. Duong*,1-4,6
}

${ }^{1}$ Research Imaging Institute, Departments of ${ }^{2}$ Radiology; ${ }^{3}$ Ophthalmology and ${ }^{4}$ Physiology; ${ }^{5}$ Laboratory Animal Resources, University of Texas Health Science Center, San Antonio, TX, USA; ${ }^{6}$ South Texas Veterans Health Care System, San Antonio, TX

\begin{abstract}
The goal of this study was to develop a clinically relevant non-human primate (baboon) stroke model and multi-parametric MRI protocols on a clinical scanner with long-term goals to better model human stroke and facilitate clinical translations of novel therapeutic strategies. Baboons were chosen because of their relatively large brain volume and that they are evolutionarily close to humans. Middle cerebral artery occlusion (MCAO) was induced using a minimally invasive endovascular approach to guide an inflatable balloon catheter into the MCA and followed by permanently or transiently inflate the balloon. Using multimodal MRI, including perfusion and diffusion imaging, the spatiotemporal dynamic evolution of the ischemic lesions in permanent and transient occlusion experiments in baboons were investigated. Perfusion-diffusion mismatch, which approximates the ischemic penumbra, was detected. In the permanent MCAO group $(n=2)$, the mean infarct volume was $29 \mathrm{ml}(17 \%$ of total brain volume) whereas in the transient MCAO group ( $\mathrm{n}=$ 2, 60 or $90 \mathrm{~min}$ of occlusion), the mean infarct volume was $15 \mathrm{ml}$ ( $9 \%$ of total brain volume). Substantial perfusiondiffusion mismatch tissue ( $\sim 50 \%$ ) was salvaged by reperfusion compared to permanent MCAO. This baboon stroke model has the potential to become a translational platform to better design clinical studies, guide clinical diagnosis and improve treatment time windows in patients.
\end{abstract}

Keywords: Penumbra, cerebral ischemia, middle cerebral artery occlusion, nonhuman primate, perfusion, diffusion, magnetic resonance imaging.

\section{INTRODUCTION}

Ischemic stroke is a major cause of death and a leading cause of long-term disability worldwide, constituting a major societal burden. One of the major goals of stroke research is to develop clinically relevant stroke models and find ways to identify viable tissue in a timely manner using noninvasive imaging methods. The ischemic penumbra, tissue at risk surrounding the initial ischemic core, is potentially salvageable if treated in a timely manner [1]. Diffusion-weighted imaging (DWI), in which contrast is based on early changes in the apparent diffusion coefficient (ADC) of water, is a powerful tool for early detection of ischemic brain injury [2]. Ischemic lesions are apparent as regions of hyperintensity in diffusion weighted images and hypointensity in apparent diffusion coefficient maps. A stroke lesion is characterized by regions of decreased ADC, and the signal intensity within this region may be quite inhomogeneous. Often there is an infarcted lesion core (ADC decrease of 30-50\% from baseline) and a hypoperfused lesion periphery (mild to moderate decrease in ADC) [3].

\footnotetext{
*Address correspondence to this author at the Research Imaging Institute, University of Texas Health Science Center at San Antonio, 8403 Floyd Curl Drive, San Antonio, TX 78229, USA; Tel: 210567 8120;

Fax: 210567 8152; E-mail: duongt@uthscsa.edu
}

Perfusion-weighted imaging (PWI) highlights brain tissue regions with abnormal or deficient cerebral blood flow (CBF). Tissue surrounding by regions of severely decreased diffusion abnormality that have a perfusion deficit, but no diffusion abnormality exhibit a phenomenon referred to as the "perfusion-diffusion mismatch", which has been shown to approximate the ischemic penumbra [4-6].

Experimental stroke models are instrumental in the development of imaging tools to identify viable tissue, to study stroke pathophysiology, and to test novel interventions under controlled experimental conditions. The availability and ease of manipulation of rodent models have greatly advanced our understanding of cerebral ischemia. While numerous treatments have been shown to be effective in rodent stroke models, none, with the exception of recombinant tissue plasimogen activator (rtPA), has proven to be effective clinically. A possible explanation is that rodent stroke models may not sufficiently reflect the complexity of human neurophysiology in stroke. The Stroke Therapy Academic Industry Roundtable (STAIR) has recommended that new stroke intervention strategies be tested on animals that are evolutionarily closer to humans, such as nonhuman primates (NHPs), before clinical trials [7]. NHP brains share more similar vascular anatomy and neurophysiological function to humans compared to other lower animal species, such as rodents. Thus, NHP stroke characteristics, in principle, should better model human stroke, providing an important preclinical test 
bed to accelerate translation of novel intervention strategies to clinical practices.

NHPs have been used as stroke models [8-15] but studies in which MRI, beyond basic anatomical imaging, was employed are sparse. Changes in perfusion, diffusion and/or fractional anisotropy in marmosets $[9,16]$, macaque [15, 17, 18] and baboon [19] stroke models have been reported. However, studies that show the perfusion-diffusion mismatch phenomenon in NHP stroke models have not been, to our knowledge, systematically investigated.

The goal of this study was to develop a baboon stroke model and multi-parametric MRI protocols on a clinical scanner with the long-term goals to better model human stroke and facilitate clinical translations of novel therapeutic strategies. Baboons were chosen because they have large brain volumes (i.e., more than twice that of macaques) with extensive cortical folding $[20,21]$ making them amenable to MRI studies. The large vessel size also facilitates endovascular approach to the MCA. A minimally invasive endovascular approach was utilized to induce stroke, which is well suited for MRI studies, in contrast to the more invasive transorbital or intracranial procedures [8]. With multimodal MRI, including perfusion and diffusing imaging, the spatiotemporal dynamic evolution of ischemic lesion in permanent and transient stroke in baboons were investigated.

\section{MATERIALS AND METHODOLOGY}

\section{Stroke Model}

Four normal female baboons (Papio hamadryas spp., 14$19 \mathrm{~kg}, 16 \pm 2 \mathrm{~kg}$ ) were studied with approval from the Institutional Animal Care and Use Committee of the University of Texas Health Science Center at San Antonio. Anesthesia was induced with ketamine (1.0-1.2 c.c., i.m.) for intubation and catheterization for fluid supplementation. Animals were mechanically ventilated and anesthetized with 1.5\%-2.0\% isoflurane in medical air during surgical cannulation of the femoral artery. End-tidal $\mathrm{CO}_{2}\left(\mathrm{ETCO}_{2}\right), \mathrm{O}_{2}$ saturation, heart rate, and respiration rate were continuously monitored during surgery.

Permanent $(\mathrm{n}=2)$ or transient $(60$ - or 90-min, $\mathrm{n}=2)$ middle cerebral artery occlusion (MCAO) was induced using minimally invasive endovascular techniques. Occlusion on the right or left MCA was determined randomly among different animals. Access to the right common femoral artery employed a micropuncture set and the Seldinger technique. A 5 Fr vascular sheath was inserted into the femoral artery over a 0.035" guide wire (Bentson, AngioDynamics, Latham, NY, USA). Selective catheterization of the internal carotid artery (ICA) was accomplished using a 5 Fr catheter (VER135, Cordis, Bridgewater, NJ, USA) over the glide wire. Selective anterior cerebral angiogram and road mapping (Siemens SIREMOBIL Compact L) were performed by injecting contrast media (Omnipaque, GE Healthcare, Buckinghamshire, UK) through the guiding catheter at the ICA. A smaller HyperGuide balloon catheter $(3 \times 10$ mm, EV3, Irvine, CA, USA) was inserted over its 0.010 ” glide wire, coaxially through the guiding catheter into the MCA. The balloon catheter was inflated to occlude the M2 segment of the MCA. Occlusion was confirmed with an anterior cerebral angiogram. The balloon catheter (which is not MRI com- patible) was deflated and withdrawn after 60 or 90 min of occlusion. After stroke surgery, all of the catheters were removed and the animal was transported to the MRI suite down the hall. Non-reperfusion was subsequently confirmed in two animals (permanent MCAO group, 60- or 90-min occlusion before withdrawing the occluder) and successful reperfusion was confirmed in the other two animals (transient MCAO group) throughout the MRI studies using magnetic resonance angiography (MRA).

The animal preparations for MRI studies in baboons have been described previously [22, 23]. Briefly, the animal was positioned supine and secured in a custom-made, MRI compatible animal holder and stabilized with ear bars, a mouth bar and padding around the head before transportation. During MRI, isoflurane was lowered to $0.8 \% \sim 1.0 \%$ and added anesthetic support achieved with vecuronium $(0.2 \mathrm{mg} / \mathrm{kg}$ bolus and $0.02 \mathrm{mg} / \mathrm{kg}$ as needed, i.v.). Rectal temperature was maintained within normal physiological ranges (37-38 ${ }^{\circ} \mathrm{C}$ ) using a feedback-regulated circulating warm-air blanket. $\mathrm{ETCO}_{2}, \mathrm{O}_{2}$ saturation, heart rate, and respiration rate were continuously monitored with an MRI-compatible physiological monitor device (Precess, InVivo, Orlando, FL, USA) during imaging. Blood gases and blood glucose were typically sampled once or twice after during stroke surgery and during MRI. All physiological parameters were maintained within normal ranges.

MRI acquisition started 30-60 min after withdrawal of the balloon occluder. One each block of MRI acquisitions was repeated every $30 \mathrm{~min}$ up to 8 hours (permanent MCAO) after occlusion or 3 hours (transient MCAO) after reperfusion. Follow-up MRIs on days 1, 3, 7, and 30 after stroke were acquired in the transient MCAO group. In the permanent MCAO group, animals were euthanized $8 \mathrm{hrs}$ post-occlusion. Permanent MCAO was confirmed by MRA periodically during MRI. In the transient MCAO group, In the transient MCAO group, one animal (60 min transient MCAO) was euthanized 30 days after stroke, and the other animal (90 min MCAO) was euthanized about 24 hrs after stroke due to degrading clinical signs, probably because of stroke lesion compressing the brain stem. In the transient MCAO group, recannalization was confirmed by MRA periodically during MRI studies.

\section{MRI Acquisition}

MRI studies were performed on a clinical 3T Siemens TIM-Trio (Siemens Medical Solutions, Erlangen, Germany) using a standard 12-channel human head coil as a receiver and the integrated circularly polarized body coil as the transmitter. A 30-min block of MRI acquisitions included anatomical (3D high-resolution T1-weighted images and T2weighted fluid-attenuated inversion recovery (FLAIR) images), perfusion (pseudo-continuous arterial spin labeling, pCASL), diffusion MRI, and MRA. Anatomical T1weighting images were acquired using the MPRAGE pulse sequence with $\mathrm{TR} / \mathrm{TE}=2100 / 3.1 \mathrm{~ms}$, flip angle $=12^{\circ}, \mathrm{TI}=$ $1100 \mathrm{~ms}, \mathrm{FOV}=16 \times 19.2 \times 19.2 \mathrm{~cm}^{3}, 1 \mathrm{~mm}$ isotropic spatial resolution and $\mathrm{NEX}=2$. T2-weighted FLAIR images were acquired with $\mathrm{TR} / \mathrm{TE} / \mathrm{TI}=4000 / 76 / 1800 \mathrm{~ms}, 1 \times 1 \times 2 \mathrm{~mm}^{3}$ spatial resolution. pCASL images were acquired using a single-shot gradient-echo EPI with TR/TE = 3500/16 ms, labeling duration $=2.1 \mathrm{sec}, 12$ contiguous slices with $4 \mathrm{~mm}$ slice 
thickness, matrix $=64 \times 64$, FOV $=12.8 \times 12.8 \mathrm{~cm}(2 \times 2 \times 4$ $\mathrm{mm}^{3}$ resolution), labeling offset $=60 \mathrm{~mm}$, and post-labeling delay $=700 \mathrm{~ms}$ as described previously [22]. Diffusionweighted MRI was acquired using TR/TE $=4700 / 89 \mathrm{~ms}$, bvalue $=0 \mathrm{~s} / \mathrm{mm}^{2}$ and $1100 \mathrm{~s} / \mathrm{mm}^{2}, 30$ directions, spin-echo EPI read-out with $2 \mathrm{~mm}$ isotropic resolution.

\section{Data Analysis}

Data were processed using custom codes written in MATLAB (The MathWorks Inc., Natick, MA, USA). Quantitative cerebral blood flow was calculated as described in Wey et al. [22]. Apparent diffusion coefficient maps were calculated as described by Meng et al. [24]. Final infarct (corrected for edema as described in [24]) was determined on T2-FLAIR images on each of their last time point before euthanasia. Lesion volumes were determined by three independent observers manually drawing region-of-interests (ROIs) based on perfusion (CBF maps) and diffusion (ADC maps) images at all time points. ROIs of the infarct, defined by the endpoint T2-FLAIR images, were used for plotting temporal progression of MRI parameters.

\section{RESULTS}

The animal physiology was maintained within normal ranges before and after MCAO as well as after recovery of the transient MCAO group. Animals regained consciousness after anesthetics were discontinued and had the ability of self-feeding within 1 hour after they were awaken. One animal in the transient MCAO (60 min occlusion) showed a minor sign of movement deficit of the affected arm while the other (90 min occlusion) showed hemiparesis of both the affected arm and leg.

Fig. (1) shows the cerebral angiogram of the brain of one animal with a permanent occlusion. Angiograms clearly show the patent right ICA (Fig. 1a, dashed arrow) and cerebral vasculature in the MCA territory. The position of the balloon was between the two radiopaque markers in the subtracted image (Fig. 1b, arrow). MCA occlusion, after balloon inflation, was angiographically confirmed by the absence of $\mathrm{x}$-ray contrast distally (Fig. 1c, circled region).

In the contralateral hemisphere, quantitative CBF and ADC remained unchanged across all time points (data not shown). Fig. (2) shows representative MRA, CBF, and ADC maps at $30 \mathrm{~min}$ post-reperfusion, and the T2-weighted FLAIR image of the brain at end-point from a transient MCAO animal (90 min MCAO). An apparent post-ischemic early hyper-perfusion, as compared with the normal hemisphere, was observed within the previously occluded brain territory in this animal, indicative of efficient recannalization. The hypointense region in the ADC map indicated the ischemic zone. At 24 hours, T2-weighted FLAIR images revealed the infarct territory.

Fig. (3) shows lesion volumes from the permanent MCAO group. Hypointense regions within the CBF and ADC maps 4.5 hrs after occlusion indicated the ischemic territories. The time courses of the lesion volume evolution (from one animal) indicated that the CBF-defined lesion remained relatively unchanged whereas the ADC-defined lesion volume, which was initially small, grew over time time to that of the CBF-defined volume. The anatomical difference between CBF and ADC abnormalities, i.e., the perfusion-diffusion mismatch, was substantial, diminished over time, and disappeared by 6 hours post-occlusion. The average final infarct volume was $\sim 29 \mathrm{~mL}$ from two animals ( $\sim 17 \%$ of total brain volume).

Fig. (4) shows the lesion volumes from the transient MCAO group. Hypointense regions in the ADC maps $1 \mathrm{hr}$ after reperfusion indicate the ischemic territories. Hyper- and hypo-perfusion, as compared with the normal hemisphere, were observed after reperfusion (Fig. 4a). The time course of the ADC-defined lesion volume (from one animal, $60 \mathrm{~min}$ MCAO) was markedly different from that of the permanent MCAO group. ADC-defined lesion volume decreased with time during the first few hours after reperfusion and peaked 1 day after stroke, and was followed by a secondary decrease on days 3 and 7, likely due to ADC renormalization. The infarct volume was defined by the endpoint. The T2weighted FLAIR image volume was slightly larger than the ADC-defined lesion volume. The final infarct volume defined by T2-weighted FLAIR was $\sim 4 \mathrm{~mL}$ in this representative animal (60 min MCAO), and $\sim 26 \mathrm{~mL}$ in the other animal of the MCAO group (90 min MCAO, Fig. 2). The averaged final infarct volume, defined by T2-weighted FLAIR, was $\sim 15 \mathrm{~mL}$ from the two animals ( 9\% of total brain vol-

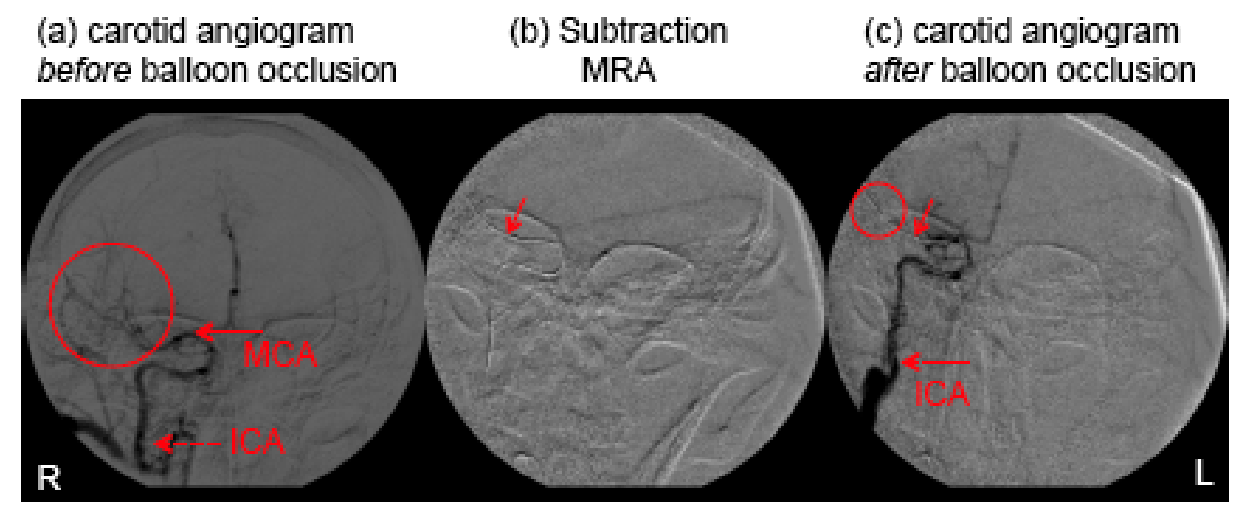

Fig. (1). Cerebral x-ray angiography (fluoroscopy) with an anterior-posterior view during stroke procedures: (a) right hemisphere cerebral angiogram with contrast injected via the right carotid artery before occlusion, (b) subtracted digital radiography showing the position of the balloon (arrow), (c) right hemisphere cerebral angiogram with contrast injected in the right carotid artery after balloon occlusion of the middle cerebral artery. 
24 hours

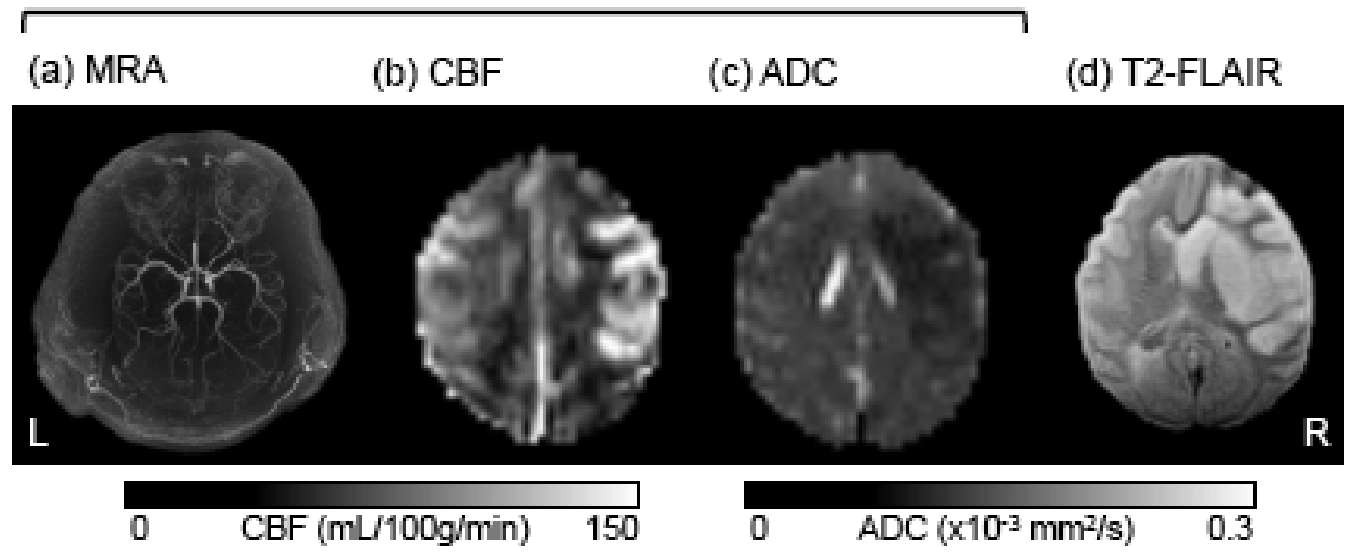

Fig. (2). A representative set of MR images from a baboon which underwent 90-min transient MCAO on the right side, including (a) MRA after reperfusion, (b) cerebral blood flow (CBF) map, (c) apparent diffusion coefficient (ADC) map, and (d) end-point T2-weighted FLAIR at 24 hours post-occlusion.

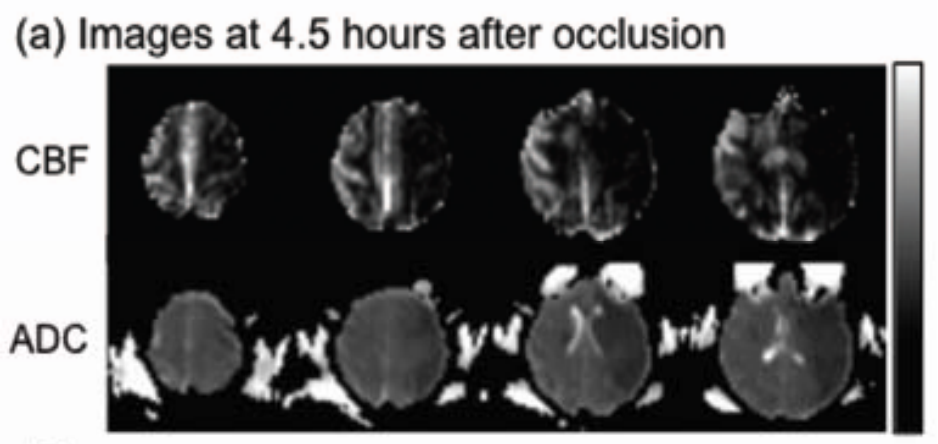

(b)

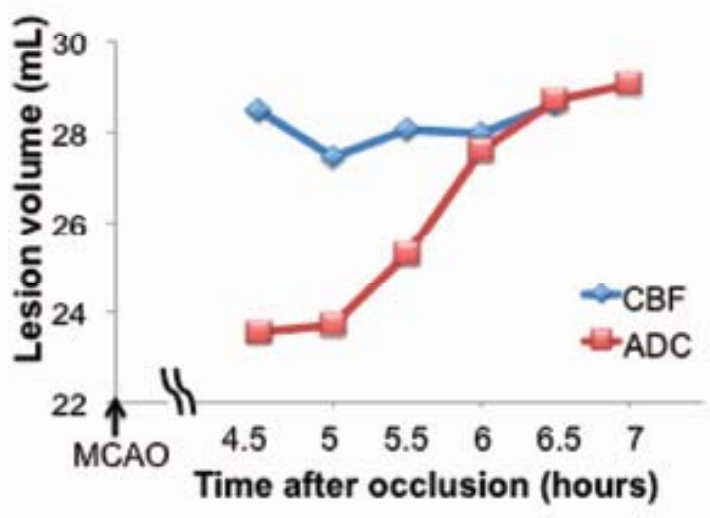

Fig. (3). Permanent MCAO: (a) cerebral blood flow (CBF, upper row) and apparent diffusion coefficient (ADC, lower row) maps of an animal which underwent permanent occlusion. (b) Temporal evolution of lesion volumes were defined by abnormal CBF and ADC. The difference between the two lines indicates the perfusion-diffusion mismatch. The grayscale bar indicates a CBF of 0 to $120 \mathrm{~mL} / 100 \mathrm{~g} / \mathrm{min}$ and an ADC of 0 to $0.3 \times 10^{-3} \mathrm{~mm}^{2} / \mathrm{sec}$.

ume), indicating that reperfusion salvaged substantial mismatch tissue compared to permanent MCAO (29mL, 17\% of total brain volume).

\section{DISCUSSION}

This study describes a minimally invasive endovascular surgical procedure to induce permanent and transient MCAO in a clinically relevant, large non-human primate (baboon) model and a multimodal MRI protocol to longitudinally characterize ischemic evolution. Perfusion-diffusion mismatch in the MR images, which approximated the ischemic penumbra, was detected. A substantial amount of the mismatched tissue was salvaged by early reperfusion compared to the permanent MCAO model.

In this baboon model, permanent MCAO, the perfusiondiffusion (PWI-DWI) mismatch detected was similar to those reported in humans and rodents. The mismatch volume progressively decreased for up to 6 hours post-occlusion, in contrast to mismatch volumes in rodent models which lasted up to 3 hours post-occlusion [25]. In humans, PWI-DWI mismatch is often detected 6-12 hours after stroke onset. The frequency of detection decreased with time [26]. The tempo- 


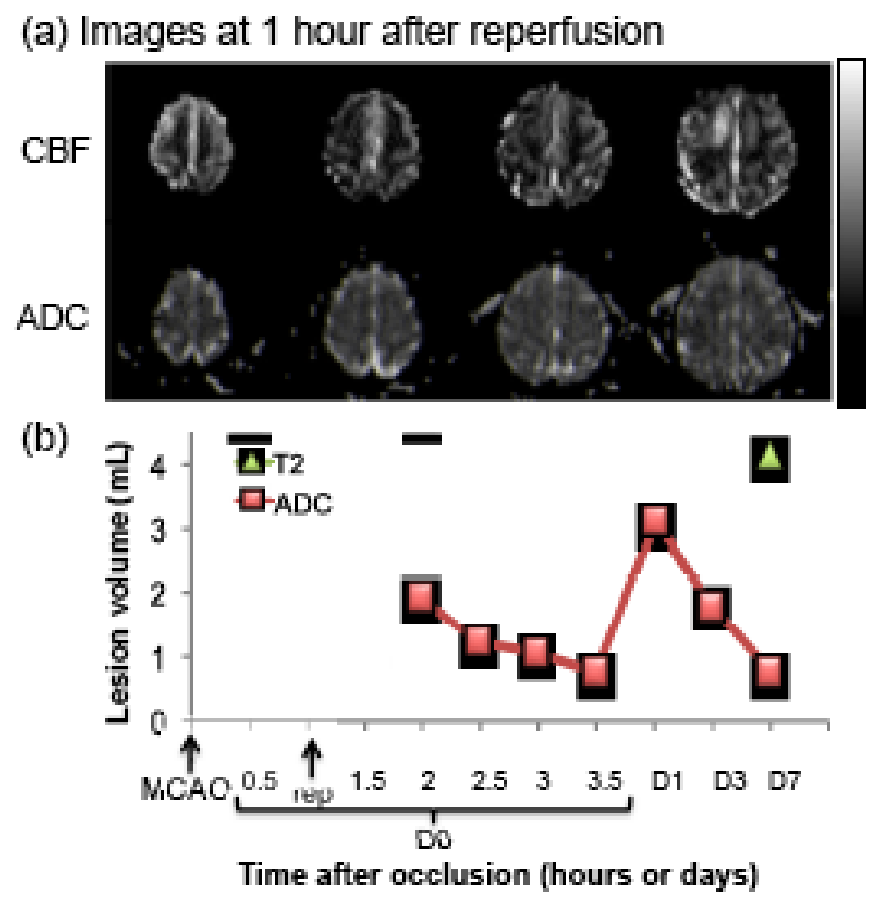

Fig. (4). Transient MCAO: (a) cerebral blood flow (CBF, upper row) and apparent diffusion coefficient (ADC, lower row) maps of an animal subjected to a 60-min transient occlusion. (b) Temporal evolution of lesion volumes were defined by CBF and ADC abnormalities. Final infarct volume, as determined by T2-weighted FLAIR MRI, is also shown. The grayscale bar indicates a CBF of 0 to $120 \mathrm{~mL} / 100 \mathrm{~g} / \mathrm{min}$ and an ADC of 0 to $0.3 \times 10^{-3} \mathrm{~mm}^{2} / \mathrm{sec}$.

ral topography of PWI-DWI mismatch in humans appeared more similar to that observed in baboons than that reported in rodents.

Early recannalization salvaged a substantial portion of tissue immediately after reperfusion when compared with those subjects with infarcts but without reperfusion. The ADC-defined lesion decreased a few hours after reperfusion but grew markedly after day 1 , consistent with the reports in rodents [24] and humans [4-6]. Although the T2-weighted FLAIR infarct volume of the transient MCAO group peaked at day 7, it was markedly smaller than that of the permanent MCAO group, indicating that reperfusion salvaged a substantial amount of tissue which provides evidence supporting the concept of the mismatch in this stroke model. Comparing our results with the published results in rodents, infarct volume typically stopped evolving by 1 or 2 days after stroke [24]. This finding further corroborates the notion that ischemic evolution in human brains appears to be more similar to that seen in the NHP model than in rodent models.

Early post-ischemic hyperperfusion was detected in the transient MCAO group but not in the permanent MCAO group. This observation suggests that this NHP model could offer an opportunity to investigate hyperperfusion in stroke. Early post-ischemic hyperperfusion, a common phenomenon in human stroke patients [27, 29], and rodent stroke model [30] is an indicator of successful reperfusion. Controversy exists regarding whether early post-ischemic hyperperfusion is beneficial or harmful [27-29]. In the transient MCAO of this study regions on with early post-ischemic hyperperfusion eventually developed into infarct, suggesting possible reperfusion injury. Additional studies with this baboon stroke model might provide insights for developing interventions against reperfusion injury.

The major shortcomings of the current study are: 1) Reproducibility of the stroke model has not yet been fully established given the small sample sizes, 2) MRI during occlusion would be helpful but unfortunately, to our knowledge, there is no commercially available balloon catheter that is MRI compatible. The balloon catheter causes MRI susceptibility artifacts and cannot be used in the magnet. The cause of permanent MCAO after withdrawal of the balloon catheter was likely due to vasospasm. Future baboon stroke studies will increase sample size to establish reproducibility, establish ADC and CBF viability thresholds, include other multimodal MRI methods (i.e. fMRI and pH MRI), investigate different MCAO durations to mimic various clinical conditions from hyperacute to chronic phase, evaluate functional reorganization in chronic stroke, and compare with immunohistology and behavioral/neurological scores. Future studies will also explore the embolic stroke model, which may better mimic clinical conditions albeit the infarct volumes produced will likely be more variable compared to the balloon occlusion approach. The NHP stroke model is more expensive than the rodent model, so rodent stroke models will remain indispensable for initial testing of novel imaging modalities and emerging therapeutic interventions.

\section{CONCLUSION}

Perfusion-diffusion mismatch, which approximates the ischemic penumbra, was detected in a clinically relevant, large non-human primate (baboon) stroke model. Reperfusion salvaged substantial amount of mismatch tissue compared to permanent stroke. These initial findings offer encouraging results to further explore the baboon stroke model 
with multimodal MRI. There are a number of dissimilarities between rodent and human brains that may lead to differences in response to identical ischemic insults. The timing, dose and other aspects of pharmacokinetics in humans could not be easily extrapolated from rodent models, which may have contributed, in part, to the negative outcomes of some clinical trials on stroke treatments. Thus, this large NHP stroke model has the potential to accelerate clinical translations of novel therapeutics and imaging tools in stroke research.

\section{ACKNOWLEDGEMENTS}

This work was supported by the American Heart Association Established Investigator Award 0940104N, National Institute of Health (NINDS R01 NS45879) and Clinical Translational Science Award's imaging supplement (parent grant UL1RR025767) to TQD. HYW is supported by a Predoctoral Fellowship (11PRE5670005) from the American Heart Association and by a Translational Science Training Grant from the Institute for Integration of Medicine and Science, University of Texas Health Science Center. The authors thank Dr. James Scribner for his initial inputs in the development of the baboon stroke model.

\section{REFERENCES}

[1] Astrup J, Siesjö BK, Symon L. Thresholds in cerebral ischemia the ischemic penumbra. Stroke 1981; 12(6): 723-5.

[2] Moseley ME, Cohen Y, Mintorovitch J, et al. Early detection of regional cerebral ischemia in cats: comparison of diffusion- and T2-weighted MRI and spectroscopy. Magn Reson Med 1990;14(2): 330-46.

[3] Kidwell CS, Alger JR, Saver JL. Beyond mismatch: evolving paradigms in imaging the ischemic penumbra with multimodal magnetic resonance imaging. Stroke 2003; 34(11): 2729-35.

[4] Baird AE, Benfield A, Schlaug G, et al. Enlargement of human cerebral ischemic lesion volumes measured by diffusion-weighted magnetic resonance imaging. Ann Neurol 1997; 41(5): 581-9.

[5] Barber PA, Davis SM, Darby DG, et al. Absent middle cerebral artery flow predicts the presence and evolution of the ischemic penumbra. Neurology 1999; 52(6): 1125-32.

[6] Schlaug G, Benfield A, Baird AE, et al. The ischemic penumbra: operationally defined by diffusion and perfusion MRI. Neurology 1999; 53(7): 1528-37.

[7] Fisher M. Recommendations for standards regarding preclinical neuroprotective and restorative drug development. Stroke 1999; 30(12): 2752-8.

[8] Fukuda S, del Zoppo GJ. Models of focal cerebral ischemia in the nonhuman primate. ILAR J 2003; 44(2): 96-104.

[9] Freret $\mathrm{T}$, Bouet $\mathrm{V}$, Toutain $\mathrm{J}$, et al. Intraluminal thread model of focal stroke in the non-human primate. J Cereb Blood Flow Metab 2008; 28(4): 786-96.

[10] Huang J, Mocco J, Choudhri TF, et al. A modified transorbital baboon model of reperfused stroke. Stroke 2000; 31(12): 3054-63.

[11] Kito G, Nishimura A, Susumu T, et al. Experimental thromboembolic stroke in cynomolgus monkey. J Neurosci Methods 2001; 105(1): 45-53.
[12] Liu XG, Branston NM, Kawauchi M, Symon L. A model of acute focal ischemia in the territory of the anterior cerebral artery in baboons. Stroke 1992; 23(1): 40-4.

[13] D'arceuil HE, Duggan M, He J, Pryor J, De Crespigny A. Middle cerebral artery occlusion in Macaca fascicularis: acute and chronic stroke evolution. J Med Primatol 2006; 35(2): 78-86.

[14] Spetzler RF, Selman WR, Weinstein P, et al. Chronic reversible cerebral ischemia: evaluation of a new baboon model. Neurosurgery 1980; 7(3): 257-61.

[15] West GA, Golshani KJ, Doyle KP, et al. A new model of cortical stroke in the rhesus macaque. J Cereb Blood Flow Metab 2009; 29(6): 1175-86.

[16] Bihel E, Pro-Sistiaga P, Letourneur A, et al. Permanent or transient chronic ischemic stroke in the non-human primate: behavioral, neuroimaging, histological, and immunohistochemical investigations. J Cereb Blood Flow Metab 2009.

[17] Liu Y, D'arceuil HE, Westmoreland S, et al. Serial diffusion tensor MRI after transient and permanent cerebral ischemia in nonhuman primates. Stroke 2007; 38(1): 138-45.

[18] Chin Y, Sato Y, Mase M, et al. Transient decrease in cerebral motor pathway fractional anisotropy after focal ischemic stroke in monkey. Neurosci Res 2010; 66(4): 406-11.

[19] Mack WJ, King RG, Hoh DJ, et al. An improved functional neurological examination for use in nonhuman primate studies of focal reperfused cerebral ischemia. Neurol Res 2003; 25(3): 280-4.

[20] Kochunov P, Davis MD. Development of structural MR brain imaging protocols to study genetics and maturation. Methods 2010; 50(3): 136-46.

[21] Leigh SR. Brain growth, life history, and cognition in primate and human evolution. Am J Primatol 2004; 62(3): 139-64.

[22] Wey H-Y, Wang DJ, Duong TQ. Baseline CBF, and BOLD, CBF, and $\mathrm{CMRO}(2)$ fMRI of visual and vibrotactile stimulations in baboons. J Cereb Blood Flow Metab 2010; 31(2): 715-24.

[23] Wey HY, Li J, Szabo CA, et al. BOLD fMRI of visual and somatosensory-motor stimulations in baboons. Neuroimage 2010; 52: $1420-7$

[24] Meng X, Fisher M, Shen Q, Sotak CH, Duong TQ. Characterizing the diffusion/perfusion mismatch in experimental focal cerebral ischemia. Ann Neurol 2004; 55(2): 207-12.

[25] Shen Q, Meng X, Fisher M, Sotak CH, Duong TQ. Pixel-by-pixel spatiotemporal progression of focal ischemia derived using quantitative perfusion and diffusion imaging. J Cereb Blood Flow Metab 2003; 23(12): 1479-88.

[26] Darby DG, Barber PA, Gerraty RP, et al. Pathophysiological topography of acute ischemia by combined diffusion-weighted and perfusion MRI. Stroke 1999; 30(10): 2043-52.

[27] Kidwell CS, Saver JL, Mattiello J, et al. Diffusion-perfusion MRI characterization of post-recanalization hyperperfusion in humans. Neurology 2001; 57(11): 2015-21.

[28] Marchal G, Young AR, Baron JC. Early postischemic hyperperfusion: pathophysiologic insights from positron emission tomography. J Cereb Blood Flow Metab 1999; 19(5): 467-82.

[29] Pan J, Konstas A-A, Bateman B, Ortolano GA, Pile-Spellman J. Reperfusion injury following cerebral ischemia: pathophysiology, MR imaging, and potential therapies. Neuroradiology 2007; 49(2): 93-102.

[30] Tanaka Y, Nagaoka T, Nair G, Ohno K, Duong TQ. Arterial spin labeling and dynamic susceptibility contrast CBF MRI in postischemic hyperperfusion, hypercapnia, and after mannitol injection. J Cereb Blood Flow Metab 2010; 31(6):1403-11. 